\title{
Le groupe affine d'une variété riemannienne compacte
}

\author{
AbDelghani ZeghiB
}

\section{Introduction.}

Le groupe d'isométries $I \operatorname{som}(M)$ d'une variété riemannienne compacte $M$ est un groupe de Lie compact. La raison de la compacité de $\operatorname{Isom}(M)$ est simplement l'équicontinuité des tous ses éléments puisqu'ils préservent tous une même distance, celle provenant de la structure riemannienne.

Mais on peut associer à la variété riemannienne $M$ d'autres groupes de transformations préservant une structure sous-jacente, mais moins fine que sa structure riemannienne.

Ainsi on peut considérer les groupes de transformations suivants: Conf $(M)$, le groupe conforme de $M$, i.e., celui des transformations conservant les angles; $\operatorname{Affin}(M)$, le groupe des transformations affines de $M$, i.e., les transformations conservant la connexion de Levi-Civita, ou de manière équivalente conservant les géodésiques paramétrées, et enfin $\operatorname{Proj}(M)$, le groupe des transformations projectives de $M$, i.e., celles préservant les géodésiques non paramétrées.

À priori, il n'y a pas de raison de penser que ces derniers groupes soient compacts. Mais comme on sait que supporter une action d'un groupe non compact (préservant une structure géométrique rigide) est une propriété très spéciale, on a toujours pensé qu'on peut lister et décrire les variétés (riemanniennes compactes) pour lesquelles, l'un des groupes précédents n'est pas compact. La première formulation de cette idée était connue sous la forme de conjecture de Lichnerowicz, dans le cas du groupe conforme. Sa solution est due à $[\mathrm{L}-\mathrm{F}]$ et $[\mathrm{Oba}]$, qui avaient démontré que seules les sphères munies de leurs métriques canoniques admettent des groupes de transformations conformes non compacts.

À notre connaissance, le cas du groupe affine n'a pas été systématiquement investi, si ce n'est par un résultat classique, probablement peu connu, et sans suite, du à K. Yano. Plus récemment, mais motivés 
par des questions de théorie ergodique, plus que par une étude générale du groupe affine, R. Zimmer puis R. Feres avaient considéré des actions affines de certains groupes discrets. Rappelons leurs résultats:

Théorèm 1.1. [Yan] Soit $M$ une variété riemannienne compacte. Alors un flot de transformations affines est isométrique. En d'autres termes, on a égalité des composantes neutres: $\operatorname{Affin}^{0}(M)=\operatorname{Isom}^{0}(M)$.

Théorèm 1.2. Soit $M$ une variété riemannienne compacte et $\Gamma$ un réseau de $S L(n, \mathbf{R}), n \geq 3$, agissant sur $M$ en respectant sa connexion de LeviCivita. Supposons que cette action ne respecte pas de métrique riemannienne. Supposons que: $\operatorname{dim} M=n$ ([Zim1]), ou $\operatorname{dim} M=n+1$ ([Fer]), alors $M$ est un tore plat.

Les approches de ces deux derniers théorèmes sont de nature respectivement analytique (globale) et ergodique.

Ici, nous proposons un argument géométrique simple, mariant les deux précédents points de vue et permettant d'élucider la structure d'une transformation affine quelconque:

Théorèm 1.3. Soit $M$ une variété riemannienne compacte. Soit $\tilde{M}=$ $\tilde{N} \times \mathbf{R}^{n}$ la décomposition de de Rham de son revêtement universel, où $\tilde{N}$ est sans facteur plat. Soit $f$ une transformation affine de $M$, i.e., un difféomorphisme affine respectant la connexion de Levi-Civita, et $\tilde{f}$ un relèvement de $f$ dans $\tilde{M}$. Alors $\tilde{f}=(g, h)$, où $g$ est une isométrie riemannienne de $\tilde{N}$ et $h$ est une transformation affine unimodulaire de $\mathbf{R}^{n}$.

Ainsi on peut écrire explicitement Affin $(M)=\operatorname{Nor}\left(\pi_{1}(M)\right) / \pi_{1}(M)$ où $\operatorname{Nor}\left(\pi_{1}(M)\right)$ est le normalisateur du groupe fondamental $\pi_{1}(M)$ dans $\operatorname{Isom}(\tilde{N}) \times \operatorname{Affin}\left(\mathbf{R}^{n}\right)$. Mais ceci cache de formidables difficultés algébriques, plus précisément relevant de la théorie des groupes discrets des groupe de Lie. Les résultats qui suivent se déduisent de manipulations de cette théorie, basées sur le théorème précédent. Le premier résultat retrouve et améliore le Théorème de Yano, qui équivaut à dire que $\Gamma(M)=\operatorname{Affin}(M) / \operatorname{Isom}^{0}(M)$ est un groupe discret. Mais lequel?

Théorèm 1.4. Soit $M$ une variété riemannienne compacte, et soit $n$ la dimension du facteur euclidien de son revêtement universel. Alors il existe un homomorphisme l : Affin $(M) \rightarrow S L^{ \pm}(n, \mathbf{R})$ (le groupe des matrices à déterminent \pm 1 ) dont l'image est un sous-groupe dénombrable de $S L^{ \pm}(n, \mathbf{R})$. Le noyau est un groupe compact, contenu dans Isom $(M)$ et 
contenant $\operatorname{Isom}^{0}(M)$, qui coïncide ainsi avec Affin ${ }^{0}(M)$. L'image de $l$ préserve un sous-groupe dénombrable $A \subset \mathbf{R}^{n}$. L'adhérence de $A$ étant cocompact dans $\mathbf{R}^{n}$ et $\operatorname{rang}(A) \leq \operatorname{dim}(M)$.

C'est bien connu, la décomposition de de Rham du revêtement universel passe mal à la variété quotient. Mais il y a certaines structures qui subsistent:

Théorèm 1.5. Soit $M$ une variété riemannienne compacte, $\tilde{M}=\tilde{N} \times \mathbf{R}^{n}$ la décomposition de de Rham de son revêtement universel, où $\tilde{N}$ est sans facteur plat. Le facteur euclidien détermine un feuilletage $\mathcal{L}_{1}$ de $M$, parallèle et à feuilles plates. Les adhérences des feuilles de $\mathcal{L}_{1}$ déterminent un feuilletage singulier $\mathcal{L}$, à feuilles compactes plates (donc à revêtement fini près des tores). Le relevé $\tilde{\mathcal{L}}$ est défini par l'action isométrique sur $\tilde{M}$ d'un groupe abelien $L$ contenant $\mathbf{R}^{n}$.

Le groupe $\operatorname{Affin}(M)$ préserve $\mathcal{L}$, et l'action déduite sur l'espace métrique $M / \mathcal{L}$ est isométrique.

Le noyau $H$ de l'homomorphisme Affin $(M) \rightarrow \operatorname{Isom}(M / \mathcal{L})$ agit affinement et fidèlement sur les feuilles génériques de $\mathcal{L}$, qui sont toutes isométriques à une même variété plate compacte.

De plus, il existe un autre sous-feuilletage $\mathcal{L}_{2}$ de $\mathcal{L}$, orthogonale à $\mathcal{L}_{1}$. Le groupe Affin $(M)$ préserve et agit isométriquement sur $\mathcal{L}_{2}$.

Voici maintenant une généralisation du Théorème de Zimmer et Feres, où la dimension de la variété est doublée:

Corollaire 1.6. Soit $\Gamma$ un réseau de $S L(k, \mathbf{R})$ agissant affinement sur une variété riemannienne compacte $M$, avec $\operatorname{dim} M<2 k$. Alors, ou bien à indice fini près l'action est isométrique, ou bien un revêtement fini de $M$ est affinement difféomorphe à un produit $N \times \mathbf{T}^{k}$. L'action de $\Gamma$ sur $N$ est finie, et celle sur $\mathbf{T}^{k}$ se déduit d'un homomorphisme $\Gamma \rightarrow S L(k, \mathbf{Z})$, à image d'indice fini. En particulier $\Gamma$ n'est pas co-compact.

Dans [Zeg], nous démontrons un résultat analogue où l'on suppose $\operatorname{dim} M \leq n+1$, mais pour une connexion qui n'est pas nécessairement riemannienne, ce qui en fera une généralisation d'un résultat de [Goe].

Le résultat suivant ne suppose aucune restriction sur la dimension.

Corollaire 1.7. Soit $\Gamma$ un réseau non co-compact d'un groupe de Lie simple de rang $\geq 2$. Supposons que $\Gamma$ agit affinement sur une variété riemannienne compacte $M$. Alors, ou bien l'action est finie, ou bien un revêtement fini de 
$M$ est affinement difféomorphe à un produit $N \times \mathrm{T}^{k}$, pour un certain entier $k$, où $\mathbf{T}^{k}$ est le tore affine euclidien de dimension $k$. L'action de $\Gamma$ sur $N$ est finie, et celle sur $\mathbf{T}^{k}$ se déduit d'un homomorphisme $\Gamma \rightarrow S L(k, \mathbf{Z})$, à noyau finie.

Nous concluons par des exemples et quelques remarques:

Théorèm 1.8. Soit $G$ le sous-groupe de $S L^{ \pm}(s+n, \mathbf{R})$ préservant chacun des facteurs $\mathbf{R}^{n}$ et $\mathbf{R}^{s}$ ainsi que la métrique euclidienne restreinte à ce dernier facteur, et préservant également un réseau $\pi$ de $\mathbf{R}^{s+n}$,

$G=\left\{A \in S L^{ \pm}(s+n, \mathbf{R}) / A(\pi)=\pi, A\left(\mathbf{R}^{n}\right)=\mathbf{R}^{n}, A\left(\mathbf{R}^{s}\right)=\mathbf{R}^{s}\right.$, et $A$ isométrique le long de $\left.\mathbf{R}^{s}\right\}$.

Alors il existe une variété riemannienne $M$ de dimension $1+s+n$ sur laquelle $G$ agit affinement et s'identifie à Affin $(M) / \operatorname{Affin}^{0}(M)$.

Remarque 1. Dans [Fer], il a été question de connexions (sans torsion) à transport parallèle borné. C'est équivalent à dire que le groupe d'holonomie est relativement compact. Ainsi, en un point fixe, le groupe d'holonomie préserve un produit scalaire en ce point. En translatant parallèlement, on définit de manière cohérente une métrique sur la variété. Par construction cette métrique est parallèle au sens de la connexion. Par unicité de la connexion de Levi-Civita, la connexion initiale est la connexion de Levi-Civita de la métrique construite. Ainsi une connexion (sans torsion) à transport parallèle borné est simplement une connexion riemannienne.

\section{Preuve du Théorème 1.3 .}

\subsection{Décomposition de de Rham.}

Soit $M$ une variété riemannienne. Pour $x \in M$, notons $\operatorname{Hol}_{M}(x)$ le groupe d'holonomie en $x$, i.e., le groupe d'isométries de $T_{x} M$ obtenues par transport parallèle le long de lacets basés en $x$. Nous notons $\operatorname{Hol}_{M}^{0}(x)$ le groupe d'holonomie restreinte, i.e., en considérant seulement des lacets homotopes à 0 .

Proposition 2.1 (Scindement de de Rham). [K-N]. Soit $M$ une variété riemannienne compacte. Alors son revêtement universel s'écrit comme un produit riemannien maximal, unique à transposition de facteurs près, $\tilde{M}=\tilde{M}_{1} \times \ldots \times \tilde{M}_{k} \times \mathbf{R}^{n}$. 
La décomposition ainsi définie de $T \tilde{M}$ est caractérisée par le fait d'être la décomposition maximale en sous-fibrés parallèles.

Soit $x \in M$ et $\tilde{x}=\left(\tilde{x}_{1}, \ldots, \tilde{x}_{k}, \tilde{y}\right)$ un relèvement dans $\tilde{M}$. Alors le groupe d'holonomie restreinte $\mathrm{Hol}_{M}^{0}(x)$ s'identifie au produit direct: $\mathrm{Hol}_{\tilde{M}_{1}}\left(\tilde{x}_{1}\right) \times$ $\ldots \times \operatorname{Hol}_{\tilde{M}_{k}}\left(\tilde{x}_{k}\right)$. De plus chaque groupe $\operatorname{Hol}_{\tilde{M}_{i}}\left(\tilde{x}_{i}\right)$ est compact et agit irréductiblement sur $T_{\tilde{x}_{i}} \tilde{M}_{i}$.

La décomposition de de Rham détermine des feuilletages parallèles de $M$, notés, $\mathcal{F}_{1}, \ldots, \mathcal{F}_{k}$ et $\mathcal{E}$ (pour le feuilletage défini par le facteur euclidien). Les groupes d'holonomies restreintes feuilletés en $x$ seront notés: $\mathrm{Hol}_{1}^{0}(x), \ldots, \mathrm{Hol}_{k}^{0}(x)$. On a donc $\mathrm{Hol}_{M}^{0}(x)=\mathrm{Hol}_{1}^{0}(x) \times \ldots \times \mathrm{Hol}_{k}^{0}(x)$.

\subsection{Début de la preuve.}

Soit $f: M \rightarrow M$ un difféomorphisme affine et $\tilde{f}: \tilde{M} \rightarrow \tilde{M}$ un relèvement de $f$ au revêtement universel. Vu sa caractérisation si ci-dessus, $\tilde{f}$ induit une permutation de la décomposition de de Rham, en particulier quitte à passer à une puissance de $f$, on peut supposer que $\tilde{f}$ respecte cette décomposition. De plus, pour $x \in M$ et $y=f x$, la dérivée $d_{x} f: T_{x} M \rightarrow T_{y} M$ établit une conjugaison entre $\operatorname{Hol}_{M}^{0}(x)$ et $\operatorname{Hol}_{M}^{0}(y)$, et par conséquent également entre les facteurs $\mathrm{Hol}_{i}(x)$ et $\mathrm{Hol}_{i}(y)$. Soit $\tau_{x y}: T_{y} M \rightarrow T_{x} M$ un transport parallèle identifiant $T_{y} M$ et $T_{x} M$. Alors $d_{x} f \tau_{x y}: T_{x} M \rightarrow T_{x} M$ est une application linéaire de $T_{x} M$, normalisant $\operatorname{Hol}_{M}^{0}(x)$, ainsi que chaque facteur $\operatorname{Hol}_{i}^{0}(x)$.

Lemme 2.2. Soit $K$ un sous-groupe compact de $O(m)$ agissant irréductiblement sur $\mathbf{R}^{m}$ et soit $A$ un élément de $G l(m, \mathbf{R})$ normalisant $K$. Alors: $A=\lambda B$, où $\lambda \in \mathbf{R}$ et $B \in O(n)$. En particulier si $\operatorname{det} A= \pm 1$, alors $A \in O(m)$.

Preuve. Soit g la transformé par $A$ de la métrique euclidienne. Elle est invariante par le groupe $K$, car ce dernier est normalisé par $A$. La métrique $g$ s'obtient à partir de la métrique euclidienne via une matrice symétrique $B$. Les espaces propres de $B$ seront invariants par $K$. Par irréductibilité, $B$ admet une seule valeur propre. En d'autres termes $g$ est multiple de la métrique euclidienne.

Corollaire 2.3. $d_{x} f$ établit une homothétie d'un rapport $\lambda_{i}(x)$ entre $T_{x} \mathcal{F}_{i}$ et $T_{f x} \mathcal{F}_{i}$. 


\subsection{Formes volumes et courbure.}

Affirmation Soit $\omega_{i}$ la forme volume du feuilletage $\mathcal{F}_{i}$. C'est une forme parallèle. Il existe une constante $\lambda_{i}$ telle que $f^{*} \omega_{i}=\lambda_{i} \omega_{i}$.

Preuve. Comme $\omega_{i}$, la forme $f^{*} \omega_{i}$ est parallèle. Elle est par ailleurs évidemment proportionnelle à $\omega_{i}$. Par parallélisme des deux formes, la constante de proportionnalité est elle même une fonction parallèle, i.e., constante.

On en déduit immédiatement:

Corollaire 2.4. Les fonctions $\lambda_{i}(x)$ ci-dessus sont eonstantes égales $\grave{a} \lambda_{i}$. Autrement dit, $d_{x} f$ établit une homothétie de rapport $\lambda_{i}$ entre $T_{x} \mathcal{F}_{i}$ et $T_{f x} \mathcal{F}_{i}$.

Affirmation On $a \lambda_{i}= \pm 1$.

Preuve. D'après ce qui précède, on peut écrire la restriction de $d f$ au sousfibré $T \mathcal{F}_{i}$ sous la forme $\lambda_{i} B_{i}$, où $B_{i}$ est un endomorphisme isométrique (i.e., conservant la métrique sur les fibres) de $T \mathcal{F}_{i}$ au dessus de $f$.

Soit $R_{i}: T \mathcal{F}_{i} \times T \mathcal{F}_{i} \times T \mathcal{F}_{i} \rightarrow T \mathcal{F}_{i}$, le tenseur courbure du feuilletage $\mathcal{F}_{i}$ (rappelons que ce dernier est géodésique). L'invariance de $R_{i}$ par la puissance $f^{n}$ de $f$ se traduit par: pour tous $X, Y$ et $Z$ sections de $T \mathcal{F}_{i}$, on a:

$$
\left.\lambda_{i}^{3 n} R_{i}\left(B_{i}^{n} X, B_{i}^{n} Y\right) B_{i}^{n} Z=\lambda_{i}^{n} B_{i}^{n}(R(X, Y) Z)\right)
$$

Donc:

$$
\left.R_{i}\left(B_{i}^{n} X, B_{i}^{n} Y\right) B_{i}^{n} Z=\lambda_{i}^{-2 n} B_{i}^{n}(R(X, Y) Z)\right)
$$

Or, les endomorphismes $B_{i}$ sont isométriques, et par suite les puissance $B_{i}^{n}$ sont uniformément bornées. Ainsi le premier membre de l'egalité précédente est uniformément (en $n$ ) borné. Le second ne le sera que si $R_{i}(X, Y) Z=0$ ou $\lambda_{i}= \pm 1$. En conclusion si $\lambda_{i}$ n'est pas égale à \pm 1 , alors le tenseur courbure $R_{i}$ est identiquement nul, i.e., les feuilles de $\mathcal{F}_{i}$ sont plates. Ceci contredit notre choix d'un facteur non euclidien.

Fin de la preuve du Théorème 1.3. Évidemment le fait que $\lambda_{i}=$ \pm 1 , pour tout $i$, signifie exactement que $\tilde{f}$ agit isométriquement sur les facteurs non euclidiens. La composante euclidienne de $\tilde{f}$ est évidemment une transformation affine de $\mathbf{R}^{n}$. Il ne reste de la preuve du Théorème 1.3 
que de montrer que cette transformation est unimodulaire. Ceci équivaut au fait que $f$ préserve le volume riemannien de $M$. Ceci se fait comme si dessus. Si $\omega$ est la forme volume de $M$ alors $f^{*} \omega=\lambda \omega$, pour une constante $\lambda$. Donc $\operatorname{Vol}(f(M))=|\lambda| \operatorname{Vol}(M)$. Comme $f$ est bijective, $\lambda= \pm 1$. La preuve de 1.3 est ainsi achevée. $\diamond$.

\section{Preuve des Théorèmes 1.4 et 1.5 .}

La preuve est une variation autour du Théorème de Bieberbach. En effet, le théorème 1.5 découle immédiatement du Théorème de Bieberbach si les projections du groupe fondamental $\pi_{1} \operatorname{sur} I \operatorname{som}\left(\mathbf{R}^{n}\right)$ et $I \operatorname{som}(\tilde{N})$ sont discretes. Ceci n'est pas toujours le cas, analysons-nous donc la situation.

\subsection{Le Théorème 1.4 .}

Soit $\operatorname{Affin}(M)$ le groupe relevé de $\operatorname{Affin}(M)$ dans $\tilde{M}$. Donc $\operatorname{Affin}(M)=A \tilde{f f i n}(M) / \pi_{1}$. Soit $\tilde{f} \in A \tilde{f f i n}(M)$. D'après $1.3, \tilde{f}=(g, h)$, avec $g \in \operatorname{Isom}(\tilde{N})$ et $h \in \mathbf{R}^{n} \times \mid S L^{ \pm}(n, \mathbf{R})$.

Soit $\tilde{l}:$ Affin $(M) \rightarrow S L^{ \pm}(n, \mathbf{R})$, l'homomorphisme qui à $\tilde{f}$ associe la partie linéaire de $h$. Cet homomorphisme ne passe pas à Affin $(M)$. En effet si $\gamma \in \pi_{1}$ alors $\tilde{f} \gamma$ et $\tilde{f}$ correspondent au même élément de $A f f i n(M)$. Mais si $\gamma=(a, b) \in I \operatorname{som}(\tilde{N}) \times I \operatorname{som}\left(\mathbf{R}^{n}\right)$ et $b=t r \in \mathbf{R}^{n} \times \mid O(n)$, alors $\tilde{l}(\tilde{f} \gamma)=\tilde{l}(\tilde{f}) r$.

Soit donc $R$ la projection de $\pi_{1}$ sur $O(n)$. Il est, ainsi que sa clôture algébrique $Z$, normalisés par $\tilde{l}\left(A \tilde{f} f i n(M)\right.$ ) (car $\pi_{1}$ est normalisé par $A \tilde{f f i n}(M))$.

On a donc besoin de définir une variante de $\tilde{l}$, un homomorphisme à valeurs dans $\operatorname{Nor}(Z) / Z$. Le groupe algébrique $Z$ s'écrit comme un produit presque direct (i.e., qui devient direct dans un revêtement fini) $Z=K \cdot T$, où $K$ est semi-simple est $T$ est un tore algébrique.

Traîtons le facteur semi-simple $K$. Le quotient $\operatorname{Nor}(K) / K$ s'identifie naturellement, au moins à indice fini près, à $\operatorname{Cent}(K)$, le centralisateur de $K$. En effet si $A$ normalise $K$, il y induit un automorphisme qu'on peut supposer interne (quitte à passer à une puissance), c'est-à-dire, égal à $A d(k)$ pour un certain $k \in K$. Donc $A k^{-1}$ centralise $K$.

Il est également facile de réaliser canoniquement pour un tore algébrique $T$, le quotient $N o r(T) / T$ comme un sous-groupe de $S L^{ \pm}(n, \mathbf{R})$ centralisant $T$. 
En définitive, on a un homomorphisme bien défini: $l:$ Affin $(M) \rightarrow$ $S L^{ \pm}(n, \mathbf{R})$, qui est non trivial sur les transformations non isométriques.

Il est trivial sur $\operatorname{Isom}^{0}(M)$ car les éléments de ce groupe agissent trivialement sur $\pi_{1}$. Donc ils agissent par conjugaison, trivialement sur $\mathbf{R}^{n}$. En effet c'est le cas de leur action sur la projection de $\pi_{1} \operatorname{sur} \operatorname{Isom}\left(\mathbf{R}^{n}\right)$ qui est un groupe assez large. Plus précisément il existe un groupe $A \subset \mathbf{R}^{n}$ dénombrable de rang $\leq \operatorname{dim} M$, et d'adhérence co-compact, qui est normalisé par Affin $(M)$. Ce ne sera rien d'autre que la projection sur $\mathbf{R}^{n}$ du groupe $\pi$ construit dans l'affirmation suivante. $\diamond$

\subsection{Le groupe $L$.}

On a $\pi_{1} \subset \operatorname{Isom}(\tilde{M})=I \operatorname{som}(\tilde{N}) \times I \operatorname{som}\left(\mathbf{R}^{n}\right)$ et $I \operatorname{som}\left(\mathbf{R}^{n}\right)=\mathbf{R}^{n} \rtimes O(n)$. Le groupe fondamental $\pi_{1}$ est co-compact dans $I \operatorname{som}(\tilde{M})$. Donc également $\pi_{1}^{0}=\pi_{1} \cap I \operatorname{som}^{0}(\tilde{M})$ est co-compact dans la composante neutre $I \operatorname{som}^{0}(\tilde{M})$, qui est égale à $\operatorname{Isom}^{0}(\tilde{N}) \times\left(\mathbf{R}^{n} \times \mid S O(n)\right)$.

Affirmation Il existe un sous-groupe de Lie abelien $L_{2}$ de Isom $(\tilde{N})$ tel que $\pi_{1}$ coupe le sous-groupe abelien $L=L_{2} \times \mathbf{R}^{n}$ en un réseau $\pi$ de $L: \pi_{1} \cap L$ est un réseau de $L$. De plus la projection de $\pi$ sur $L_{2}$ est dense, et $L$ est normalisé par $\pi_{1}: \gamma L=L \gamma$, pour tout $\gamma \in \pi_{1}$.

Preuve. Soit $N i l$ le nilradical, i.e., le plus grand sous-groupe normal nilpotent, de $\operatorname{Isom}^{0}(\tilde{N})$. Le nilradical de $\operatorname{Isom}^{0}(\tilde{M})$ est alors $N i l \times \mathbf{R}^{n}$.

Pour étudier la trace de $\pi_{1}^{0}$ (où de $\pi_{1}$, cela revient au même) sur ce nilradical, on dispose d'un théorème d'Auslander et Wang qui généralise de loin le théorème de Bieberbach [Rag]. Son applicabilité présuppose que le groupe de Lie $\operatorname{Isom}^{0}(\tilde{M})$ ne contient pas de sous-groupe semi-simple compact qui soit un facteur presque direct (au sens que son action par conjugaison sur les autres facteurs soit finie).

Pour contourner cette difficulté, écrivons $\operatorname{Isom}^{0}(\tilde{N})=K \cdot G$ où $K$ est le facteur compact semi-simple et presque direct maximal. Une conséquence directe de théorème de Wang et Auslander est qu'alors, la trace de $\pi_{1}^{0}$ sur $K \cdot N i l \times \mathbf{R}^{n}$ est co-compact. En effet il suffit de tout quotienter par $K$. La projection ainsi obtenue transforme un réseau en un réseau, car $K$ est compact.

En fait, toujours d'après le même type de résultats sur les réseaux de groupes de Lie, les mêmes faits d'intersection co-compacte, sont vrais en remplaçant le nilradical, par son centre [Rag]. En conclusion, il existe un sous-groupe de Lie normal abelien $C$ de $I \operatorname{som}^{0}(\tilde{N})$ tel que $\pi_{1} \cap\left(K \cdot C \times \mathbf{R}^{n}\right)$ soit co-compact dans $K \cdot C \times \mathbf{R}^{n}$. 
Comme l'intersection de $\pi_{1}$ avec $K$ est fini, on peut s'arranger pour qu'elle soit triviale. Donc $\pi=\pi_{1} \cap\left(K \cdot C \times \mathbf{R}^{n}\right)$ s'injecte dans $C \times \mathbf{R}^{n}$ et est en particulier abelien. La projection de $\pi$ sur $K$ est également un sousgroupe abelien de $K$, dont on note $K^{\prime}$, la composante neutre de l'adhérence. On prendra alors $L=K^{\prime} \cdot C \times \mathbf{R}^{n}$. Il est clair que $\pi_{1}$ normalise $L$.

\subsection{Fin de la preuve de 1.5 .}

Les orbites de $L$ définissent un feuilletage (singulier) $\tilde{\mathcal{L}}$ de $\tilde{M}$, qui passe en un feuilletage $\mathcal{L}$ de $M$, car $\pi_{1}$ normalise $L$ (l'action de $L$ ne passe pas!). Les feuilles de $\mathcal{L}$ sont toutes compactes, car elles sont toutes revêtues par $L / \pi$. Elles sont évidemment invariantes par les feuilletages $\mathcal{L}_{1}$ et $\mathcal{L}_{2}$ déterminés de la même manière respectivement à partir des groupes $L_{1}=\mathbf{R}^{n}$ et $L_{2}$ (ci-dessus).

Sur toute feuille de $\mathcal{L}$, ces feuilletages se déduisent par projection, des deux feuilletages linéaires définis par $L_{1}$ et $L_{2}$ sur le tore $L / \pi$. En particulier, car par construction $\pi . L_{1}$ est dense dans $L$, les feuilles de $\mathcal{L}$ ne sont rien d'autre que les adhérences des feuilles de $\mathcal{L}_{1}$.

On avait directement construit $\mathcal{L}$ comme orbite d'une action isométrique sur $\tilde{M}$, ce qui en fait un feuilletage riemannien (singulier) par excellence. En fait, il est vrai en général que les adhérences des feuilles d'un feuilletage riemannien, déterminent un feuilletage, également riemannien [Mol]. Toutefois, l'énoncé analogue pour les feuilletages parallèles est faux: les adhérences des feuilles d'un feuilletages parallèle est certes, riemannien, pas nécessairement parallèle. Dans notre cas, les feuilles de $\tilde{\mathcal{L}}$ et par suite celles de $\mathcal{L}$ sont plates. En effet une feuille de $\tilde{\mathcal{L}}$ est munie d'une action isométrique transitive d'un groupe abelien. Donc le fait que $\mathcal{L}$ soit parallèle contredit la définition de $\mathcal{L}_{1}$ (comme facteur euclidien maximal de $\tilde{M}$ ) sauf si $\mathcal{L}=\mathcal{L}_{1}$. En fait les feuilles de $\mathcal{L}$ ne sont généralement pas géodésiques (voir la preuve de 1.8).

On tire directement de 1.3 que $\operatorname{Affin}(M)$ respecte $\mathcal{L}$ et agit isométriquement sur le quotient $M / \mathcal{L}$.

Soit $f$ un élément de $\operatorname{Affin}(M)$ agissant trivialement sur $M / \mathcal{L}$, et $\tilde{f}$ un relèvement de $f$ dans $\tilde{M}$. Donc pour tout $x \in \tilde{M}, \tilde{f}\left(\left(\pi_{1} \cdot L\right) x\right)=\left(\pi_{1} \cdot L\right) x$. En composant avec un élément $\gamma \in \pi_{1}$, on peut supposer que: $\tilde{f} L x=L x$, $\forall x \in \tilde{M}$. Supposons que $\tilde{f}$ agit trivialement sur une feuille $L x$, alors $\tilde{f}$ est une isométrie. En effet comme dans $1.3, \tilde{f}=(g, h)$. Si $\tilde{f}$ est triviale sur $L x=\left(L_{2} \times \mathbf{R}^{n}\right) x$, alors nécessairement la composante $h$ est triviale.

Pour un feuilletage singulier $\mathcal{L}$, une isométrie $f$ qui le respecte, et qui est triviale sur l'espace quotient ainsi que sur une feuille générique, est triviale (il 
suffit de remarquer que $f$ agit trivialement sur les géodésiques orthogonales à cette feuille). $\diamond$

4. Preuves de $1.6,1.7$ et 1.8 .

\subsection{Preuve du Corollaire 1.6.}

Soit $\Gamma \subset S L(k, \mathbf{R})$ un réseau agissant sur $M$, avec $\operatorname{dim} M<2 k$. Écrivons comme ci-dessus: $\tilde{M}=\tilde{N} \times \mathbf{R}^{n}$. On a donc au moins l'une des deux possibilités: $n<k$, ou $\operatorname{dim} \tilde{N}<k$.

Supposons $n<k$. D'après 1.4, on a une représentation $l:$ Affin $(M) \rightarrow$ $S L(n, \mathbf{R})$. D'après le Théorème de supper-rigidité de Marguilis (voir [Zim2]), la restriction de $l$ à $\Gamma$ est fini $(\operatorname{car} n<k)$. Donc d'après 1.4, à indice fini près, $\Gamma$ est contenue dans $\operatorname{Isom}^{0}(M)$.

Supposons maintenant que $k<n$. Un théorème de Marguilis [Zim3] affirme alors que tout homomorphisme de $\Gamma$ dans un groupe compact agissant sur une variété de dimension $<n$ est fini. Ainsi à indice fini près, $\Gamma$ est contenu dans le noyau $H$ de l'homomorphisme de 1.5.

Une petite manipulation permet de transformer l'action de $\Gamma$ sur une feuille de $\mathcal{L}$, à la situation suivante. Le groupe $\Gamma$ agit sur $\mathbf{R}^{s} \times \mathbf{R}^{n}$ en respectant chacun des facteurs, ainsi que la métrique sur $\mathbf{R}^{s}$ et de plus en préservant un réseau $\pi$. Comme $s<k$, par le même Théorème de Marguilis, à indice fini près l'action de $\Gamma$ sur $\mathbf{R}^{s}$ est trivial.

Appliquons le Théorème de supper-rigidité de Marguilis [Zim2]. La répresentation de $\Gamma$ dans $S L(n, \mathbf{R})$ est la restriction d'une représention $\phi^{\prime}$ : $S L(k, \mathbf{R}) \rightarrow S L(n, \mathbf{R})$ (on a ici exclu le cas où l'action de $\Gamma$ est isométrique, donc la représentation de $\Gamma$ dans $S L(n, \mathbf{R})$ n'est pas bornée). Quitte à conjuguer par un élément de $S L(n, \mathbf{R})$, on peut supposer que $\phi^{\prime}$ est de la forme: $\phi^{\prime}(A)=(1, \phi(A))$ où 1 est l'identité dans $\mathbf{R}^{n-d}$ et $\phi: S L(k, \mathbf{R}) \rightarrow$ $S L(d, \mathbf{R})$ est une représentation sans vecteur (globalement) fixe. Ainsi $A \in \Gamma$ agit sur $\mathbf{R}^{s} \times \mathbf{R}^{n}=\mathbf{R}^{s+n-d} \times \mathbf{R}^{d}$ par $(a, b) \rightarrow(a, \phi(A) b)$. L'observation clé est:

$$
(a, b) \in \pi \Longrightarrow(0, \phi(A) b-b) \in \pi
$$

Comme $d<2 n$, la représentation $\phi$ est à automorphisme de $S L(k, \mathbf{R})$ près, la représentation canonique (i.e., l'identité) de $S L(k, \mathbf{R})$. Toutefois, pour pouvoir appliquer le même argument à la section suivante, dans le reste de la preuve, on n'utilisera ce fait que pour en déduire l'égalité: $d=k$. 
Le corollaire 1.6 qu'on est en traîn de démontrer équivaut au fait que le scindement $\mathbf{R}^{s+n-d} \times \mathbf{R}^{d}$ induit un scindement de $\pi$. Ceci équivaut à son tour au fait que la projection $\pi^{\prime}$ de $\pi$ sur $\mathbf{R}^{d}$, ainsi que l'intersection $\pi^{\prime \prime}=\pi \cap \mathbf{R}^{d}$, sont des réseaux (discrets) de $\mathbf{R}^{d}$. L'observation ci dessus donne:

$$
A \in \Gamma \Longrightarrow(\phi(A)-1)\left(\pi^{\prime}\right) \subset \pi^{\prime \prime}
$$

En particulier, $(\phi(A)-1)\left(\pi^{\prime}\right)$ est discret.

Pour montrer que $\pi^{\prime}$ est un réseau, il suffit de montrer qu'il est discret. Supposons qu'il ne l'est pas. Soit $V$ la composante neutre de la fermeture de $\pi^{\prime}$. Donc $\pi^{\prime} \cap V$ est dense dans $V$. Évidemment, $V$ est invariant par $\phi$. Donc, pour $A \in \Gamma,(\phi(A)-1)\left(\pi^{\prime} \cap V\right)$ est un sous-groupe discret de $V$. Par densité de $\pi^{\prime} \cap V$ dans $V$, ceci entraîne que $\phi(A)$ est trivial sur $V$. Ainsi l'adionde $\phi$ est triviale sur $V$, ce qui contredit notre définition ci-dessus de $\phi$.

Supposons maintenant que $\pi^{\prime \prime}$ n'est pas un réseau de $\mathbf{R}^{d}$ et notons $V$ son enveloppe linéaire. D'après ce qui précéde, $(\phi(A)-1)\left(\mathbf{R}^{d}\right) \subset V$, pour tout $A \in \Gamma$. Ceci s'étend à tous les éléments $A \in S L(k, \mathbf{R})$ (par densité algébrique de $\Gamma$ dans $S L(k, \mathbf{R})$ ). Ainsi la représentation quotient de $\phi$ sur $\mathbf{R}^{d} / V$ est triviale. Mais une représentation sans vecteur fixe, d'un groupe de Lie semi-simple n'admet pas de facteur trivial. En conclusion, $\pi^{\prime \prime}$ est un réseau de $\mathbf{R}^{d}$. $\diamond$

\subsection{Preuve de 1.7 .}

La preuve est exactement la même que ci-dessus, sachant que toujours d'après Marguilis (voir [Zim3]), tout homomorphisme d'un réseau non cocompact de rang supérieur dans un groupe compact, est fini. En d'autres termes, il n'y a pas de restriction de dimension dans le cas non co-compact. $\diamond$.

\subsection{Preuve du Théorème 1.8 .}

Considérons le réseau $\mathbf{Z} \times \pi$ de $\mathbf{R}^{1+s+n}$. L'idée est de tordre la métrique euclidienne sur ce dernier espace vectoriel pour ainsi briser certaines symétries, de telle façon que le facteur euclidien dans la décomposition de de Rham de la nouvelle métrique soit $\mathbf{R}^{n}$. Munissons $\mathbf{R} \times \mathbf{R}^{s} \times \mathbf{R}^{n}$ des coordonnées $(t, x, y)$, et d'une métrique de la forme: $g=d t^{2}+f(t) d x^{2}+d y^{2}$, avec $f$ une fonction sur $\mathbf{R}$ de période exactement 1 . Ainsi cette métrique est invariante par les translations entières le long de l'axe des $t$. Le groupe 
d'isométries de $g$ est $\mathbf{Z} \times I \operatorname{som}\left(\mathbf{R}^{s+n}\right)$, agissant naturellement. Le facteur euclidien dans la décomposition de de Rham est simplement $\mathbf{R}^{n}$. Pour le voir, remarquons par exemple que l'introduction de la fonction $f$ a permis de briser le caractère géodésique des orbites de $\mathbf{R}^{s}$ et le caractère isométrique du flot de $\partial / \partial t$. On prendra pour variété riemannienne $M$, le tore $\mathbf{R}^{1+s+n} / \mathbf{Z} \times \pi$, muni de la métrique quotient. On vérifie directement que $\operatorname{Affin}(M)=\mathbf{T}^{s+n} \times \mid G$.

\section{References.}

[Fer] R. Feres: "Connections preserving actions of lattices in $S L(n, \mathbf{R})$ ", Israel. J. Math. (2) 135 (1992), 1-21.

[Goe] E. Goetze: "Connection preserving actions of connected and discrete Lie groups", J. Diff. Geom. 40 (1994), 595-620.

[K-N] S. Kobayashi, K. Nomizu: "Foundations of differential geometry, volume1", Interscience, New York, 1963.

[L-F] J. Lelong-Ferrand: "Transformations conformes et quasi conformes des variétés riemanniennes: application à la démonstration d'une conjecture de A. Lichnerowicz", C.R.Acad.Sci. Paris, Série A, 269 (1969), 583-586.

[Oba] M. Obata: "The conjectures about conformal transformations", J. Diff. Geom. 6 (1971), 247-258.

[Rag] M.S. Raghunathan: "Discrete subgroups of Lie groups", SpringerVerlag, New-York (1972).

[Yan] K. Yano "On harmonic and Killing vector fields", Ann. Math. 55 (1952), 38-45.

[Zeg] A. Zeghib: "Sur les actions affines des groupes discrets", Ann. Inst. Fourier, 47(1997), 1-45.

[Zim] R. Zimmer: " On connection-preserving actions of discrete linear groups", Erg. Th. Dynam. Systems, 6 (1986), 639-644.

[Zim2] R. Zimmer: "Ergodic theory and semisimple Lie groups", Birkhäuser, 1984, Boston. 
[Zim3] R. Zimmer: "Lattices in semisimple groups and invariant geometric structures on compact manifolds", Discrete Groups in Geometry and Analysis (R. Howe, editor), Birkhäuser, Boston (1987), 152-210.

RECeived August 9, 1995.

CNRS, UMPA, ENS LYON, 46, ALLÉE D'ITALIE 69364 LYON CEDEX 7 FRANCE

ZEGHIB@UMPA.ENS-LYON.FR 